\title{
Are Onconeural Antibodies a Clinical Phenomenology in Paraneoplastic Limbic Encephalitis?
}

\author{
Hongliang Zhang, ${ }^{1,2}$ Chunkui Zhou, ${ }^{1,3}$ Limin $\mathrm{Wu}^{1,4}{ }^{1,4}$ Fengming Ni, ${ }^{5}$ Jie Zhu, $^{1,2}$ and Tao Jin ${ }^{1}$ \\ ${ }^{1}$ Department of Neurology, The First Bethune Hospital of Jilin University, Jilin University, Xinmin Street 71, Changchun 130021, China \\ ${ }^{2}$ Department of Neurobiology, Care Sciences and Society, Karolinska Institute, Novum, Plan 5, 14186 Stockholm, Sweden \\ ${ }^{3}$ Department of Neurology, The Second Part of the First Hospital, Jilin University, Lequn Street, Changchun 130021, China \\ ${ }^{4}$ Neuroprotection Research Laboratory, Massachusetts General Hospital, Harvard Medical School, Charlestown, MA 02129, USA \\ ${ }^{5}$ Department of Radiotherapy, The First Bethune Hospital of Jilin University, Xinmin Street 71, Changchun 130021, China
}

Correspondence should be addressed to Tao Jin; taotao_jin2004@yahoo.com.cn

Received 21 March 2013; Revised 31 May 2013; Accepted 4 July 2013

Academic Editor: Jessica Teeling

Copyright ( 2013 Hongliang Zhang et al. This is an open access article distributed under the Creative Commons Attribution License, which permits unrestricted use, distribution, and reproduction in any medium, provided the original work is properly cited.

\begin{abstract}
Paraneoplastic neurological syndromes (PNSs) occur in patients with cancer and can cause clinical symptoms and signs of dysfunction of the nervous system that are not due to a local effect of the tumor or its metastases. Most of these clinical syndromes in adults are associated with lung cancer, especially small cell lung cancer (SCLC), lymphoma, and gynecological tumors. The finding of highly specific antibodies directed against onconeural antigens has revolutionized the diagnosis and promoted the understanding of these syndromes and led to the current hypothesis of an autoimmune pathophysiology. Accumulating data strongly suggested direct pathogenicity of these antibodies. The field of PNS has expanded rapidly in the past few years with the discovery of limbic encephalitis associated with glutamic acid decarboxylase (GAD) 65, the voltage (VGKC-gated potassium channel) complex, the methyl (N-NMDA-D-aspartate), alpha-amino-3-hydroxy-5-methyl-4-isoxazolepropionic acid (AMPA), and gamma aminobutyric acid (GABA) (B) receptors, and so forth. Despite this, the clinical spectrum of these diseases has not yet been fully investigated. The clinical importance of these conditions lies in their frequent response to immunotherapies and, less commonly, their association with distinctive tumors. This review provides an overview on the pathogenesis and diagnosis of PNS, with emphasis on the role of antibodies in limbic encephalitis.
\end{abstract}

\section{An Overview of Paraneoplastic Neurological Syndromes}

The idea that neural cells can be the target of autoimmune responses mediated by antibodies is still not well recognized in the medical community [1]. Paraneoplastic neurological syndromes (PNSs) are rare dysfunctions of the nervous system in patients with cancer, which are not due to a local effect of the tumor or its metastases. Most of these clinically defined syndromes in adults are associated with lung cancer, especially small cell lung cancer (SCLC), lymphoma, or gynecological tumors. Antibodies directed against onconeural antigens are frequently detected in patients with PNS. So far, these antibodies have been thought to be the only markers of the disease and not to play a role in the pathophysiology. However, the recent description of antibodies directed against membrane receptors or ion channels and playing a pathogenic role has challenged this concept. In case of antibodies targeting intracellular onconeural antigens, patients almost always harbor a tumor; some tumors might be found several years after the onset of neurological symptoms. However, it is not the case in the patients with antibodies targeting surface antigens (ion channels, receptors, or receptor associated proteins).

The reported incidence of PNS varies greatly since most estimates are from referral centers and not from populationbased studies [2]. Paraneoplastic sensory neuropathy is probably the most common (3-7 per 1000 cancer diagnoses), 
followed closely by paraneoplastic encephalitis (3 per 1000) and cerebellar degeneration (2 per 1000) [3]. A rough classification of PNS is illustrated in Table 1 [4].

\section{Limbic Encephalitis: An Increasingly Recognized Entity Belonging to PNS}

The limbic system of brain comprises hippocampus, amygdala, hypothalamus, corpus mamillare, fornix, and gyrus cinguli (the Papez circuit) and is responsible for cognition, affect, and autonomic regulation. Limbic encephalitis was described for the first time by Brierley and colleagues in 1960 [5]. It is characterized by subacute onset (from days to several months) of short-term memory loss, disorientation, seizures, confusion, behavioral disturbance, psychiatric symptoms, and altered consciousness suggestive of involvement of the limbic system [6]. Less frequently, patients can have delusional thoughts and paranoid ideation [7], and some patients may have hyponatremia.

In the last decades, limbic encephalitis has been extensively investigated. According to the current knowledge, all types of limbic encephalitis fall into one of two main categories, infectious or autoimmune etiology. Infectious limbic encephalitis is caused by direct invasion of the brain by infectious agents, usually viruses, whereas autoimmune limbic encephalitis is caused by the individual's autoimmune reaction against itself. The current review will center on autoimmune limbic encephalitis and its clinical characteristics. Of note is that although the etiology was historically considered paraneoplastic, limbic encephalitis may also arise from nonparaneoplastic mechanisms, that is, autoimmune processes independent of malignancy. The clinical presentations are quite similar in the two groups. Prodromal flulike symptoms may point to a nonparaneoplastic etiology, whereas smoking and weight loss suggest a paraneoplastic etiology [8]. The difficulty in differentiating the two categories stems from the fact that in $60 \%$ to $70 \%$ of paraneoplastic cases, neurological symptoms precede the detection of the tumor $[9,10]$.

Established diagnosis of this syndrome after exclusion of infective and toxic disorders should prompt the initiation of immunotherapy [11]. The following investigations may aid an accurate diagnosis: analysis of cerebrospinal fluid (CSF), electroencephalogram (EEG), magnetic resonance imaging (MRI), positron emission tomography (PET), and detection of onconeural antibodies in the CSF and/or serum. CSF usually shows lymphocytic pleocytosis, increased protein concentration, and oligoclonal bands. Regardless of the type of clinical presentation, EEG is almost always abnormal, typically revealing focal or generalized slow wave abnormalities or epileptic form discharges in the temporal lobes [12]; T2-weighted or fluid-attenuated inversion recovery (FLAIR) MRI may show hyperintense signals of the medial temporal lobes, although other sites of lesions can also be detected (Figure 1); ${ }^{18} \mathrm{~F}$-fluorodeoxyglucose (FDG) positron emission tomography (PET) may detect hypermetabolism in the medial temporal lobes, even when MRI is normal [12]; various antibodies may be present in serum and CSF. The information provided by the combination of clinical, EEG,

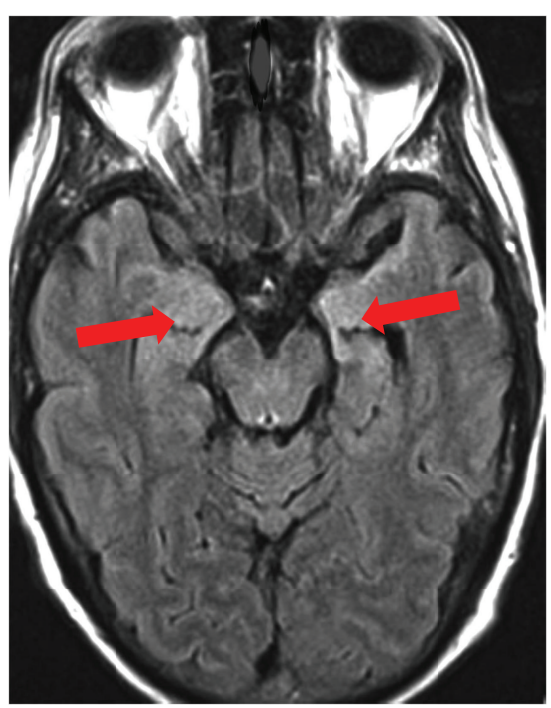

FIGURE 1: MRI FLAIR of a patient with limbic encephalitis and positive NMDAR antibodies in the CSF. Increased signal intensity is seen in the bilateral medial temporal lobes and hippocampi.

MRI, and CSF routine studies suggests the diagnosis of limbic encephalitis in most patients with a classic presentation of the syndrome [12]. It is not mandatory that all investigations show pathological features, and not all cases of limbic encephalitis have typical MRI findings. However, if EEG, MRI, and CSF analyses are all normal, the diagnosis of limbic encephalitis is highly unlikely [8]. The diagnostic criteria for limbic encephalitis are listed in Table 2 [13], and the differential diagnoses of limbic encephalitis are summarized in Table 3.

Clinical characteristics of the different types of limbic encephalitis significantly vary according to the antibody type. NMDAR encephalitis often presents with cognitive and behavioral abnormalities. Because psychiatric symptoms are early and prominent, it is not rare for patients to be treated with antipsychotic drugs at onset. Subsequently, characteristic features develop, including movement disorders (orofacial dyskinesia, dystonia), seizures, speech disorder, autonomic dysfunction, central hypoventilation, catatonia, and depressed level of consciousness $[8,11]$. Patients with PNS and LGI1-antibodies usually present with classic limbic encephalitis but may show some specific features, such as hyponatremia, rapid eye movement (REM), sleep behavioral disorders, or characteristic tonic seizures. Factually, the concept that limbic encephalitis is an inflammatory disorder strictly limited to anatomic regions of the limbic system is inaccurate. In this regard, these nonrestricted inflammatory boundaries are the rule rather than the exception, particularly when the limbic encephalitis is paraneoplastic. This is evidenced by many pathologic studies that have shown inflammatory infiltration distant from the limbic system. In these patients, a careful clinical evaluation almost always reveals signs of involvement of other areas of the nervous system that may remain mild or become more prominent than the symptoms of limbic dysfunction. For example, PNS in 
TABLE 1: Classification of paraneoplastic neurological syndromes.

\begin{tabular}{l}
\hline Central nervous system \\
Limbic encephalitis \\
Encephalomyelitis \\
Brainstem encephalitis \\
Stiff-person syndrome \\
Opsoclonus-myoclonus \\
Subacute cerebellar degeneration \\
Paraneoplastic visual syndromes \\
Cancer-associated retinopathy \\
Melanoma-associated retinopathy \\
Paraneoplastic optic neuropathy \\
Motor neuron syndromes \\
Subacute motor neuronopathy \\
Other motor neuron syndromes \\
\hline Peripheral nervous system \\
Acute sensorimotor neuropathy \\
Subacute sensory neuronopathy \\
Chronic sensorimotor neuropathy \\
Subacute autonomic neuropathy \\
Paraneoplastic peripheral nerve vasculitis \\
\hline Neuromuscular junction and muscle \\
Myasthenia gravis \\
Lambert-Eaton syndrome \\
Acutymyositis/dermatomyositis necrotizing myopathy \\
Cachectic myopathy \\
Neuromyotonia \\
\hline
\end{tabular}

many patients with anti-Hu antibodies may start as limbic encephalitis that often evolves to encephalomyelitis with dorsal root ganglionitis.

\section{Tumors That Are Associated with Limbic Encephalitis}

In PNS, $50 \%$ to $80 \%$ of the patients present with neurological symptoms of PNS prior to diagnosis of tumors [15]. The associated tumors in PNS are a lung cancer in 50$60 \%$ of patients, usually SCLC (40-55\%), and the associated tumor is a testicular germ cell tumor in $20 \%$ of patients. Other associated tumors include breast cancer, thymoma, Hodgkin's lymphoma, and teratomas [2]. In paraneoplastic limbic encephalitis, the most common tumors and corresponding antibodies are SCLC (anti-Hu, anti-CRMP5, and anti-amphiphysin), testicular cancer (anti-Ma2), thymoma (anti-CRMP5), and breast cancer (anti-amphiphysin) [16]. In men younger than the age of 50 years with anti-Ma2 antibodies, limbic encephalitis is almost always associated with testicular germ cell tumors, which however can be microscopic and difficult to detect.

As one of the classical PNS, limbic encephalitis can be diagnosed within less than 5 years before cancer is detected
[14]. Removal of the tumor is critical for neurologic improvement or stabilization of symptoms in PNS. Therefore, tumor should be screened in patients with limbic encephalitis.

\section{Antibodies Commonly Detected in Limbic Encephalitis}

Tumor immunologists introduced the term "onconeural" antibodies to designate antibodies that target antigens present in neuroectodermal tissues and tumors [17]. These antibodies are unambiguously demonstrated by standardized tests, associated with limited subsets of tumors, and are present in several PNS types [1]. Since the 1980s, various onconeural antibodies have been discovered, which can serve as biomarkers for classic paraneoplastic syndromes [18]. Classical limbic encephalitides with temporal lobe seizures are associated with onconeural antibodies directed against the intracellular antigens. Onconeural antibodies are found in about $60 \%$ of the patients with paraneoplastic limbic encephalitis. The most frequent related antibodies are anti- $\mathrm{Hu}$, anti-Ma2 (with or without Mal), anti-amphiphysin, and anti-CRMP5. The majority of patients with anti-Hu antibodies have symptoms also suggestive of the dysfunction of areas of the nervous system outside the limbic system.

In recent years, the spectrum of chronic inflammatory brain diseases characterized by the presence of antigenspecific antibodies in serum and CSF has greatly expanded. Many patients with paraneoplastic limbic encephalitis previously characterized as "seronegative" have in fact antibodies against cell surface antigens. Recent studies indicated that most cases previously considered "seronegative" have, in fact, antibodies against surface antigens [19]. More and more cases such as glutamic acid decarboxylase (GAD) 65 antibody encephalitis [20], the voltage-gated potassium channel (VGKC) complex antibody encephalitis [21] (including LGI1 and Caspr2 antibodies), N-methyl-Daspartate receptor (NMDAR) antibody encephalitis [22], alpha-amino-3-hydroxy-5-methyl-4-isoxazolepropionic acid receptor (AMPAR) [23], and gamma aminobutyric acid receptor $\mathrm{GABA}(\mathrm{B})$ antibody encephalitis [24] are recognized. In a few years, the number of onconeural antibodies described in PNS has increased dramatically. Antibodies to the components of VGKCs, NMDARs, AMPARs, GABA(B), mGluR5 receptor, and glycine receptors (GlyRs) can be identified in patients and are associated with various clinical presentations, such as limbic encephalitis and complex and diffuse encephalopathies $[23,25,26]$. These diseases can be associated with tumors, but some of them are nonparaneoplastic, and antibody assays can help with the diagnosis. The identification of these new antibodies (cell surface antigen associated) has allowed recognition of a syndrome with clinical and radiological features indistinguishable from "classic limbic encephalitis." The course of the newly identified syndromes tends to be less severe and it is often possible to achieve complete recovery with prompt immunomodulatory treatment. The most representative condition is LGI1encephalitis, previously known as limbic encephalitis with VGKC complex antibodies [27, 28]. 
TABLE 2: Diagnostic criteria of paraneoplastic limbic encephalitis.

Criteria by Gultekin et al. [13]

Pathological demonstration of limbic encephalitis, or all 4 of the following.

(1) Symptoms of short-term memory loss, seizures, or psychiatric symptoms suggesting involvement of the limbic system

(2) $<4$ yr between the onset of neurological symptoms and the cancer diagnosis

(3) Exclusion of metastasis, infection, metabolic and nutritional deficits, stroke, and side-effects of therapy that may cause limbic encephalopathy

(4) At least one of the following:

(a) CSF with inflammatory findings

(b) MRI FLAIR or T2 unilateral or bilateral temporal lobe hyperintensities

(c) EEG with epileptic or slow activity focally involving the temporal lobes

Criteria by the Paraneoplastic Neurological Syndrome Euronetwork [14]

All 4 of the following items are met.

(i) Subacute onset (days or up to $12 \mathrm{wk}$ ) of seizures, short-term memory loss, confusion, and psychiatric symptoms

(ii) Neuropathologic or radiologic evidence (MRI, SPECT, PET) of involvement of the limbic system

(iii) Exclusion of other possible etiologies of limbic dysfunction

(iv) Demonstration of a cancer within $5 \mathrm{yr}$ of the diagnosis of neurologic symptoms or the development of classic symptoms of limbic dysfunction in association with a well-characterized paraneoplastic antibody (Hu, Ma2, CRMP5, amphiphysin, Ri)

These antibodies are directed against two categories of antigens: (1) intracellular antigens ( $\mathrm{Hu}, \mathrm{Ma} 2, \mathrm{CRMP5}$, amphiphysin, etc.) and (2) cell surface antigens (the VGKC complex, NMDAR, AMPARs, GABABRs, mGluR5 receptor, GlyRs, etc.). Whereas the disorders related to the first category of antibodies are associated with cancer (lung, testis, etc.), prominent brain infiltrates of cytotoxic $\mathrm{T}$ cells, and limited response to treatment, the disorders related to the second category of antibodies are associated less frequently with cancer (thymoma, teratoma), seem to be antibody mediated, and respond significantly better to immunotherapy. These two antibodies have in common the association with idiopathic or paraneoplastic limbic encephalitis [23, 24]. Seven out of $15(47 \%)$ patients with limbic encephalitis associated with GABA (B) receptor antibodies had an underlying tumor, usually an SCLC [24]. In limbic encephalitis associated with AMPAR antibodies, the frequency of cancer was $64 \%$, with SCLC being the most common type, followed by thymoma and breast cancer [23]. These patients have a better prognosis than those with antibodies against intracellular proteins [29, 30]. Table 4 summarizes the common antibodies against onconeural antigens detected in PNS and their potentially associated tumors.

\section{Do Antibodies Play a Pathogenic Role in Limbic Encephalitis?}

A cancer-stimulated immune response that cross-reacts with neural tissue-onconeural immunity-is considered the principal pathologic mechanism for PNS [31]. Some cancer cells express proteins that are normally restricted to the nervous system. For example, when serum from a patient with limbic encephalitis was incubated with the patient's cancer cells and with a rat's brain tissue, antibody fixation to the same Ma proteins on both neurons and cancer cells could be observed [31]. Pathological examination of the nervous system showed loss of neurons in affected areas of the nervous system with inflammatory infiltration by CD4+ T helper cells and $\mathrm{B}$ cells in the perivascular spaces and cytotoxic CD8+ T cells in the interstitial spaces [32-34]. Examination of CSF frequently demonstrates pleocytosis, intrathecal synthesis of IgG, and oligoclonal bands, supporting an inflammatory or immune-mediated etiology.

The discovery of paraneoplastic antineuronal antibodies resulted in the general belief that these are immune-mediated disorders triggered by onconeural antigens expressed by tumor cells. Despite the clear clinical evidence that many of the syndromes described earlier are antibody mediated, there is lack of direct evidence showing that these antibodies are pathogenic in PNS. Support for a pathogenic role of antibodies comes from the fact that the target paraneoplastic antigens are expressed both in the tumors and in the affected regions of the nervous system. Furthermore, the size of tumors is usually small and they are heavily infiltrated with inflammatory cells. Interestingly, spontaneous remissions of carcinoma may occur at the time of neurological presentation $[35,36]$. One study even found more limited disease distribution and better oncologic outcome in SCLC patients with paraneoplastic antibodies [37].

There are studies on the effects of the serum or CSF $\operatorname{IgG}$ antibodies on the neuronal function in cultured cells $[22,23,38]$ or on brain slices, but the transfer of clinical or electrophysiological evidence of disease to experimental animals by either systemic or intrathecal injection has not yet been reported, with the exception of mGluR1-Ab in paraneoplastic cerebellar degeneration [39] and reports of GAD-65 or amphiphysin antibodies $[40,41]$. In some PNSs, circumstantial evidence suggests that T-cell-mediated mechanisms play a major pathogenic role [42]. It has been suggested that the most important determinant of the underlying immunopathogenesis and responsiveness to immunosuppression is the antibody type and level of the affected individual, which may determine the response to treatment $[1,18,43]$. 
TABLE 3: Differential diagnoses of limbic encephalitis.

\begin{tabular}{|c|}
\hline Infectious disorders \\
\hline Herpes simplex virus encephalitis \\
\hline Neurosyphilis \\
\hline Progressive multifocal leukoencephalopathy \\
\hline Rabies \\
\hline Creutzfeldt-Jakob disease \\
\hline Metabolic disorders \\
\hline $\begin{array}{l}\text { Metabolic encephalopathy (uremic, hepatic, Cushing } \\
\text { syndrome, etc.) }\end{array}$ \\
\hline Wernicke-Korsakoff syndrome \\
\hline Hashimoto's encephalopathy \\
\hline Systemic autoimmune disorders \\
\hline Sjögren syndrome \\
\hline Systemic lupus erythematosus \\
\hline Antiphospholipid syndrome \\
\hline Malignancies \\
\hline Lymphoma \\
\hline Glioma \\
\hline Gliomatosis cerebri \\
\hline Degenerative disorders \\
\hline Alzheimer's disease \\
\hline Lewy-body dementia \\
\hline Frontotemporal dementia \\
\hline Others \\
\hline Stroke with posterior cerebral artery involvement \\
\hline Central nervous system vasculitis \\
\hline Temporal lobe epilepsy \\
\hline Nonconvulsive status epilepticus \\
\hline Transient global amnesia \\
\hline Acute demyelinating encephalomyelitis \\
\hline Posterior reversible encephalopathy syndrome \\
\hline Intoxication (alcohol, lithium, etc.) \\
\hline Alcohol withdrawal syndrome \\
\hline Psychiatric disorder \\
\hline
\end{tabular}

Specifically, striking differences have been found between disorders with antibodies against intracellular antigens versus those to neural surface antigens. Disorders with antibodies to intracellular antigens are considered poorly responsive to immunotherapy $[18,20]$ and may be mediated by cytotoxic $\mathrm{T}$ cells $[18,34]$. On the other hand, disorders associated with antibodies against cell surface antigens, such as the VGKCcomplex or NMDAR, often respond well to treatment [20, 44].

Some laboratory evidence supports the role of pathogenic B-cell responses in limbic encephalitis. NMDAR antibodies from patients have been shown to decrease the numbers of
NMDAR in postsynaptic dendrites of cultured hippocampal neurons. One study suggested that anti-Hu antibodies induced apoptosis when applied to cultures of neuroblastoma or mesenteric cells [45]. There is also evidence, however, pointing to that paraneoplastic limbic encephalitis may be T-cell mediated, as Hu-specific T cells have been found in the blood and CSF [46], and there are cytotoxic infiltrates of $\mathrm{T}$ cells in the brain and tumor of the patients with anti-Hu antibodies-associated encephalomyelitis [47].

A pathogenic role could only be proven for those paraneoplastic antibodies that are directed against easily accessible antigens located on the cell surface. In these disorders, indirect lines of evidence support the view that the cellular immune responses against these antigens are responsible for the neurological damage $[46,48,49]$. The relative contribution of the cellular and humoral immunity to the clinical and pathological manifestations has not been displayed. The paraneoplastic antibodies may, in these cases, be surrogate markers for T-cell activation [50]. Elevated CD8/CD3 ratios in diseases were associated with antibodies to intracellular antigens and suggested a cytotoxic T-cell-driven pathomechanism. In diseases with antibodies to surface antigens, this finding supports a B-cell-related pathomechanism, with evidence of a complement-mediated pathogenesis in patients with VGKC-complex antibodies. Interestingly, this immunopathogenic dichotomy parallels other autoimmune disorders such as polymyositis and dermatomyositis, which have a predominant T-cell- and antibody-mediated pathogenesis, respectively [51]. These observations may contribute to a rational choice in immunotherapies for these disorders [52]. A totally different mechanism seems at work in paraneoplastic cerebellar degeneration in Hodgkin's lymphoma because the target antigens of the associated anti-Tr and antimGluR1 antibodies are not expressed in Hodgkin's tumor tissue [53]. Dysregulation of the immune response in Hodgkin's lymphoma and an etiologic role for viral infections have been postulated in this disorder [53].

Thus far, it is still unclear whether antibody-mediated PNS, for example, VGKC complex antibody-associated limbic encephalitis, is driven by serum or intrathecal antibodies. The absolute concentrations of antibodies against a certain onconeural antigen are usually higher in serum than in the CSF. Moreover, antibodies are not always detectable in the CSF. Ideally, both serum and CSF samples should be sent for antibody testing, but their relative utility in followup of patients is under debate. Intrathecal synthesis of IgG and oligoclonal bands can help pointing to an immune-mediated disorder before the results of specific antibodies can be obtained, but the oligoclonal bands are not always present at onset or even thereafter, and whether their presence is evidence for ongoing pathology or merely a secondary epiphenomenon is not yet clear. The intrathecal synthesis of antibodies can actually be assessed by the calculation of the amount of specific antibodies in the CSF relative to the total CSF IgG and by comparison with similar calculations in the serum. The ratio represents intrathecal synthesis and is often higher in some PNS. In favor of a role for systemic rather than intrathecal antibodies, animal experiments have shown that certain regions of the brain, that is, the hippocampus 
TABLE 4: The common antibodies detected in PNS and their associated tumors.

\begin{tabular}{|c|c|c|}
\hline Antibodies & PNS & Associated tumors \\
\hline \multicolumn{3}{|c|}{ Antibodies against intracellular antigens } \\
\hline Anti-Hu & $\begin{array}{l}\text { sensory neuronopathy, LE, BSE, } \\
\text { encephalomyelitis }\end{array}$ & SCLC \\
\hline Anti-Yo & SCD & Gynecological cancer \\
\hline Anti-Ri & Opsoclonus-myoclonus, BSE & Breast cancer \\
\hline Anti-Ma2 & BSE, LE & Testis cancer, SCLC, breast cancer \\
\hline Anti-CRMP5 & $\begin{array}{l}\text { SCD, chorea, myelitis, LE, sensory } \\
\text { neuronopathy, optic neuritis }\end{array}$ & SCLC, thymoma \\
\hline Anti-amphiphysin & SPS, myelitis, SCD, sensory neuronopathy & SCLC, breast cancer \\
\hline Anti-GAD-65 & SPS, myelitis & SCLC, breast cancer \\
\hline Anti-SOX-1 & LEMS & SCLC \\
\hline \multicolumn{3}{|c|}{ Antibodies against cell surface onconeural antigens } \\
\hline Anti-VGCC & SPS, LEMS & SCLC \\
\hline Anti-VGKC complex & LE & SCLC, thymoma \\
\hline Anti-NMDA receptor & LE & Teratoma \\
\hline Anti-AMPA receptor & $\mathrm{LE}$ & SCLC, breast cancer, thymoma \\
\hline Anti-AQP-4 & NMO spectrum disorders & SCLC, breast cancer, thymoma \\
\hline Anti-GABA-B receptor & LE & SCLC \\
\hline Anti-CAR & Retinopathy & SCLC, melanoma, gynecological cancer \\
\hline Anti-contactin-associated protein 2 & Morvan syndrome & Thymoma \\
\hline Anti-AchR/MuSK/RyR/Titin & MG & Thymoma \\
\hline
\end{tabular}

AMPA: amino-3-hydroxyl-5-methyl-4-isoxazole-propionate; AQP-4: aquaporin 4; CAR: cancer-associated retinopathy; CRMP5: collapsin response mediator protein 5; GABA-B: gamma-aminobutyric acid B; GAD-65: glutamic acid decarboxylase 65; LE: limbic encephalitis; LEMS: Lambert-Eaton myasthenic syndrome; MG: myasthenia gravis; NMDA: N-methyl-D-aspartate; NMO: neuromyelitis optica; SCD: subacute cerebellar degeneration; SCLC: small cell lung cancer; SPS: stiff-person syndrome; VGCC: voltage-gated calcium channel; VGKC: voltage-gated potassium channel.

and the hypothalamus, seem to be particularly vulnerable, and it is notable that limbic encephalitis with VGKC-complex (LGI1 and Caspr2) antibodies and anti-NMDAR encephalitis usually start with symptoms originating from the temporal lobe cortex, even though the target antigens are present much more widely in the CNS. The former usually affects hippocampus, amygdala, and anterior temporal cortex, whereas the latter usually affects hippocampus, cerebral cortex, basal ganglia, and thalamus [54]. Until recently, only 50\% of patients with limbic encephalitis and SCLC were found antibody positive, usually harboring anti-Hu antibodies or, less frequently, other onconeural antibodies [29].

Immunopathological analysis of various antibody-associated limbic encephalitis may help elucidate the underlying immunopathogenic mechanisms, whereas unfortunately there has been a lack of laboratory data [52]. Why is limbic encephalitis reversible in patients with NMDAR antibodies that are in frequent association with ovarian teratoma [44, 55]? Furthermore, how does one classify those patients with GAD-65 antibodies that are not paraneoplastic in origin, who suffer from limbic encephalitis or chronic temporal lobe epilepsy [20]?

An important issue is that a positive report for any wellcharacterized onconeural antibody has to be assessed according to the clinical setting. All these antibodies, particularly those associated with SCLC, can be found in the patients with cancer without PNS [56]. Therefore, one should still rule out other potential causes of the neurological syndrome that is being evaluated. Up to $16 \%$ of patients with SCLC without PNS have low titers of $\mathrm{Hu}$ antibodies, whereas in the patients with PNS and $\mathrm{Hu}$ antibodies, the titers are substantially higher [37].

\section{Treatments of Limbic Encephalitis}

The basic principles of paraneoplastic limbic encephalitis therapy are resection of the tumor or oncological treatment [10]. When a patient with tumor is found in association with a possible paraneoplastic disorder, removal of the tumor is critical for neurologic improvement or stabilization of symptoms. Antibodies against onconeural antigens are sensitive and should prompt an extensive tumor screening in antibody-positive patients. In the patients with limbic encephalitis associated with ion channel/receptor antibodies, immunosuppressive or immunomodulatory treatment is promising to improve the disease. Limbic encephalitides with antibodies against intracellular onconeural antigens do not normally respond to immunosuppressive treatment; only tumor therapy may stabilize the syndrome. Treatment of PNS still remains difficult. Anti-Hu-antibody-positive patients do not normally respond to immunosuppressive treatment. The only therapy that stabilizes these patients is perhaps the 
TABLE 5: Immunotherapies for limbic encephalitis.

\begin{tabular}{l}
\hline Acute phase of the disease \\
First-line therapies \\
High-dose corticosteroids \\
Intravenous immunoglobulins \\
Plasma exchange \\
Second-line therapies \\
Rituximab \\
Cyclophosphamide \\
Maintenance therapy \\
Steroids \\
Azathioprine \\
Mycophenolate
\end{tabular}

tumor treatment itself $[16,57]$. In other PNSs associated with defined onconeural antibodies, only a few patients have beneficial effects after treatment [58]. Most patients with limbic encephalitis and ion channel receptor antibodies also respond to immunosuppressive or immunomodulatory treatment $[22,23,27,59]$. Patients with limbic encephalitis and antibodies against cell surface antigens such as VGKC or NMDAR often respond to immunotherapies, such as corticosteroids, intravenous immunoglobulin (IVIG), or plasma exchange. Other therapy regimens that might be of relevance are rituximab, cyclophosphamide, and azathioprine [60]. In limbic encephalitis patients with intracellular antibodies, Ma2-positive patients may respond better to immunosuppression than patients with anti-Hu or anti-CRMP5 antibodies [61]. Immunotherapies for limbic encephalitis have been summarized in Table 5.

\section{Some Conclusions and More Questions}

This field of immune-mediated CNS diseases is exciting but also challenging. Ideally, antibody testing should be performed using internationally validated procedures so that the diagnosis can be made and treatments started as soon as possible in the hope of restoring health, limiting hospitalization, and optimizing outcomes. Systematic studies of the treatments are needed in order to establish the best practice. Experience with the recently described antibodies, with exception of those against NMDAR, is still relatively small. Therefore, their inclusion in one particular group of the proposed classification must be viewed with caution until more cases are described. Good clinical-immunological correlations are crucial to define the clinical syndrome that most likely associates with a particular antibody.

The so-far identified antibodies might only be the tip of the iceberg, with antibodies to other membrane ion channels or receptors awaiting recognition in future. Even now, the range of clinical features exhibited by patients with VGKC, NMDAR, aquaporin (AQP) 4, or GlyR antibodies is wide and includes most aspects of the nervous system. Researchers in this field must provide good clinical descriptions of the case series associated with the antibodies they study. This approach will help clinicians identify the clinical syndromes and make a rational decision on which antibodies to request. Moreover, more effective methods are required for the detection of onconeural antibodies [62].

\section{Acknowledgments}

The work was supported by Grants from the National Natural Science Foundation of China (no. 81241147) and the China Scholarship Council (no. 2008102056).

\section{References}

[1] F. Graus, A. Saiz, and J. Dalmau, "Antibodies and neuronal autoimmune disorders of the CNS," Journal of Neurology, vol. 257, no. 4, pp. 509-517, 2010.

[2] T. Braik, A. T. Evans, M. Telfer, and S. McDunn, "Paraneoplastic neurological syndromes: unusual presentations of cancer. A practical review," American Journal of the Medical Sciences, vol. 340, no. 4, pp. 301-308, 2010.

[3] U. Nath and R. Grant, "Neurological paraneoplastic syndromes," Journal of Clinical Pathology, vol. 50, no. 12, pp. 975980, 1997.

[4] J. W. De Beukelaar and P. A. Sillevis Smitt, "Managing paraneoplastic neurological disorders," Oncologist, vol. 11, no. 3, pp. 292305, 2006.

[5] J. B. Brierley, J. A. N. Corsellis, R. Hierons, and S. Nevin, "Subacute encephalitis of later adult life. Mainly affecting the limbic areas," Brain, vol. 83, no. 3, pp. 357-368, 1960.

[6] J. A. N. Corsellis, G. J. Goldberg, and A. R. Norton, "limbic encephalitis' and its association with carcinoma," Brain, vol. 91, no. 3, pp. 481-496, 1968.

[7] R. Koide, T. Shimizu, K. Koike, and J. Dalmau, "EFA6A-like antibodies in paraneoplastic encephalitis associated with immature ovarian teratoma: a case report," Journal of Neuro-Oncology, vol. 81, no. 1, pp. 71-74, 2007.

[8] C. A. Vedeler and A. Storstein, "Autoimmune limbic encephalitis., Acta neurologica Scandinavica, no. 189, pp. 63-67, 2009.

[9] S. R. Samarasekera, A. Vincent, J. L. Welch, M. Jackson, P. Nichols, and T. D. Griffiths, "Course and outcome of acute limbic encephalitis with negative voltage-gated potassium channel antibodies," Journal of Neurology, Neurosurgery and Psychiatry, vol. 78, no. 4, pp. 391-394, 2007.

[10] Y. Ohta, I. Nagano, D. Niiya et al., "Nonparaneoplastic limbic encephalitis with relapsing polychondritis," Journal of the Neurological Sciences, vol. 220, no. 1-2, pp. 85-88, 2004.

[11] C. G. Bien and C. E. Elger, "Limbic encephalitis: a cause of temporal lobe epilepsy with onset in adult life," Epilepsy and Behavior, vol. 10, no. 4, pp. 529-538, 2007.

[12] N. D. Lawn, B. F. Westmoreland, M. J. Kiely, V. A. Lennon, and S. Vernino, "Clinical, magnetic resonance imaging, and electroencephalographic findings in paraneoplastic limbic encephalitis," Mayo Clinic Proceedings, vol. 78, no. 11, pp. 1363-1368, 2003.

[13] S. H. Gultekin, M. R. Rosenfeld, R. Voltz, J. Eichen, J. B. Posner, and J. Dalmau, "Paraneoplastic limbic encephalitis: neurological symptoms, immunological findings and tumour association in 50 patients," Brain, vol. 123, part 7, pp. 1481-1494, 2000.

[14] F. Graus, J. Y. Delattre, J. C. Antoine et al., "Recommended diagnostic criteria for paraneoplastic neurological syndromes," Journal of Neurology, Neurosurgery and Psychiatry, vol. 75, no. 8, pp. 1135-1140, 2004. 
[15] F. Graus and J. Dalmau, "Paraneoplastic neurological syndromes: diagnosis and treatment," Current Opinion in Neurology, vol. 20, no. 6, pp. 732-737, 2007.

[16] F. Graus, F. Keime-Guibert, R. Reñe et al., "Anti-Hu-associated paraneoplastic encephalomyelitis: analysis of 200 patients," Brain, vol. 124, no. 6, pp. 1138-1148, 2001.

[17] R. C. Seeger, P. M. Zeltzer, and S. A. Rayner, "Onco-neural antigen: a new neural differentiation antigen expressed by neuroblastoma, oat cell carcinoma, Wilms' tumor, and sarcoma cells," Journal of Immunology, vol. 122, no. 4, pp. 1548-1555, 1979.

[18] J. Dalmau and M. R. Rosenfeld, "Paraneoplastic syndromes of the CNS," The Lancet Neurology, vol. 7, no. 4, pp. 327-340, 2008.

[19] F. Graus, A. Saiz, M. Lai et al., "Neuronal surface antigen antibodies in limbic encephalitis: clinical-immunologic associations," Neurology, vol. 71, no. 12, pp. 930-936, 2008.

[20] M. P. Malter, C. Helmstaedter, H. Urbach, A. Vincent, and C. G. Bien, "Antibodies to glutamic acid decarboxylase define a form of limbic encephalitis," Annals of Neurology, vol. 67, no. 4, pp. 470-478, 2010.

[21] S. R. Irani, S. Alexander, P. Waters et al., "Antibodies to Kv1 potassium channel-complex proteins leucine-rich, glioma inactivated 1 protein and contactin-associated protein-2 in limbic encephalitis, Morvan's syndrome and acquired neuromyotonia," Brain, vol. 133, no. 9, pp. 2734-2748, 2010.

[22] J. Dalmau, A. J. Gleichman, E. G. Hughes et al., "Anti-NMDAreceptor encephalitis: case series and analysis of the effects of antibodies," The Lancet Neurology, vol. 7, no. 12, pp. 1091-1098, 2008.

[23] M. Lai, E. G. Hughes, X. Peng et al., "AMPA receptor antibodies in limbic encephalitis alter synaptic receptor location," Annals of Neurology, vol. 65, no. 4, pp. 424-434, 2009.

[24] E. Lancaster, M. Lai, X. Peng et al., "Antibodies to the GABAB receptor in limbic encephalitis with seizures: case series and characterisation of the antigen," The Lancet Neurology, vol. 9, no. 1, pp. 67-76, 2010.

[25] A. Boronat, L. Sabater, A. Saiz, J. Dalmau, and F. Graus, "GABAB receptor antibodies in limbic encephalitis and antiGAD-associated neurologic disorders," Neurology, vol. 76, no. 9, pp. 795-800, 2011.

[26] E. Lancaster, E. Martinez-Hernandez, M. J. Titulaer et al., "Antibodies to metabotropic glutamate receptor 5 in the Ophelia syndrome," Neurology, vol. 77, no. 18, pp. 1698-1701, 2011.

[27] A. Vincent, C. Buckley, J. M. Schott et al., "Potassium channel antibody-associated encephalopathy: a potentially immunotherapy-responsive form of limbic encephalitis," Brain, vol. 127, no. 3, pp. 701-712, 2004.

[28] M. Lai, M. G. M. Huijbers, E. Lancaster et al., "Investigation of LGI1 as the antigen in limbic encephalitis previously attributed to potassium channels: a case series," The Lancet Neurology, vol. 9, no. 8, pp. 776-785, 2010.

[29] S. Alamowitch, F. Graus, M. Uchuya, R. Reñé, E. Bescansa, and J. Y. Delattre, "Limbic encephalitis and small cell lung cancer. Clinical and immunological features," Brain, vol. 120, no. 6, pp. 923-928, 1997.

[30] L. Bataller, K. A. Kleopa, G. F. Wu, J. E. Rossi, M. R. Rosenfeld, and J. Dalmau, "Autoimmune limbic encephalitis in 39 patients: Immunophenotypes and outcomes," Journal of Neurology, Neurosurgery and Psychiatry, vol. 78, no. 4, pp. 381-385, 2007.

[31] L. Bataller and J. Dalmau, "Paraneoplastic neurologic syndromes," Neurologic Clinics, vol. 21, no. 1, pp. 221-247, 2003.
[32] D. Denny-Brown, "Primary sensory neuropathy with muscular changes associated with carcinoma," Journal of Neurology, Neurosurgery \& Psychiatry, vol. 11, no. 2, pp. 73-87, 1948.

[33] W. C. Jean, J. Dalmau, A. Ho, and J. B. Posner, "Analysis of the IgG subclass distribution and inflammatory infiltrates in patients with anti-Hu-associated paraneoplastic encephalomyelitis," Neurology, vol. 44, no. 1, pp. 140-147, 1994.

[34] F. Bernal, F. Graus, À. Pifarré, A. Saiz, B. Benyahia, and T. Ribalta, "Immunohistochemical analysis of anti-Hu-associated paraneoplastic encephalomyelitis," Acta Neuropathologica, vol. 103, no. 5, pp. 509-515, 2002.

[35] R. B. Darnell and L. M. DeAngelis, "Regression of smallcell lung carcinoma in patients with paraneoplastic neuronal antibodies," The Lancet, vol. 341, no. 8836, pp. 21-22, 1993.

[36] T. Byrne, W. P. Mason, J. B. Posner, and J. Dalmau, "Spontaneous neurological improvement in anti-Hu associated encephalomyelitis," Journal of Neurology Neurosurgery and Psychiatry, vol. 62, no. 3, pp. 276-278, 1997.

[37] F. Graus, J. Dalmau, R. Reñé et al., "Anti-Hu antibodies in patients with small-cell lung cancer: association with complete response to therapy and improved survival," Journal of Clinical Oncology, vol. 15, no. 8, pp. 2866-2872, 1997.

[38] E. G. Hughes, X. Peng, A. J. Gleichman et al., "Cellular and synaptic mechanisms of anti-NMDA receptor encephalitis," Journal of Neuroscience, vol. 30, no. 17, pp. 5866-5875, 2010.

[39] P. Sillevis Smitt, A. Kinoshita, B. De Leeuw et al., "Paraneoplastic cerebellar ataxia due to autoantibodies against a glutamate receptor," New England Journal of Medicine, vol. 342, no. 1, pp. 21-27, 2000.

[40] C. Geis, A. Weishaupt, S. Hallermann et al., "Stiff person syndrome-associated autoantibodies to amphiphysin mediate reduced GABAergic inhibition," Brain, vol. 133, no. 11, pp. 31663180, 2010.

[41] M. Manto, M. Laute, M. Aguera, V. Rogemond, M. Pandolfo, and J. Honnorat, "Effects of anti-glutamic acid decarboxylase antibodies associated with neurological diseases," Annals of Neurology, vol. 61, no. 6, pp. 544-551, 2007.

[42] J. Dalmau, H. S. Gultekin, and J. B. Posner, "Paraneoplastic neurologic syndromes: pathogenesis and physiopathology," Brain Pathology, vol. 9, no. 2, pp. 275-284, 1999.

[43] A. Vincent, S. R. Irani, and B. Lang, "The growing recognition of immunotherapy-responsive seizure disorders with autoantibodies to specific neuronal proteins," Current Opinion in Neurology, vol. 23, no. 2, pp. 144-150, 2010.

[44] J. Dalmau, E. Lancaster, E. Martinez-Hernandez, M. R. Rosenfeld, and R. Balice-Gordon, "Clinical experience and laboratory investigations in patients with anti-NMDAR encephalitis," The Lancet Neurology, vol. 10, no. 1, pp. 63-74, 2011.

[45] R. De Giorgio, M. Bovara, G. Barbara et al., "Anti-HuD-induced neuronal apoptosis underlying paraneoplastic gut dysmotility," Gastroenterology, vol. 125, no. 1, pp. 70-79, 2003.

[46] M. L. Albert, L. M. Austin, and R. B. Darnell, "Detection and treatment of activated $\mathrm{T}$ cells in the cerebrospinal fluid of patients with paraneoplastic cerebellar degeneration," Annals of Neurology, vol. 47, no. 1, pp. 9-17, 2000.

[47] R. Voltz, J. Dalmau, J. B. Posner, and M. R. Rosenfeld, “Tcell receptor analysis in anti-Hu associated paraneoplastic encephalomyelitis," Neurology, vol. 51, no. 4, pp. 1146-1150, 1998.

[48] B. Benyahia, R. Liblau, H. Merle-Beral et al., "Cell-mediated autoimmunity in paraneoplastic neurological syndromes with anti-Hu antibodies," Annals of Neurology, vol. 45, no. 2, pp. 162$167,1999$. 
[49] M. Tanaka, K. Tanaka, S. Tokiguchi, K. Shinozawa, and S. Tsuji, "Cytotoxic T cells against a peptide of Yo protein in patients with paraneoplastic cerebellar degeneration and anti-Yo antibody," Journal of the Neurological Sciences, vol. 168, no. 1, pp. 28-31, 1999.

[50] Z. Yu, T. J. Kryzer, G. E. Griesmann et al., "CRMP-5 neuronal autoantibody: marker of lung cancer and thymoma-related autoimmunity," Annals of Neurology, vol. 49, no. 2, pp. 146-154, 2001.

[51] K. Arahata and A. G. Engel, "Monoclonal antibody analysis of mononuclear cells in myopathies. I: Quantitation of subsets according to diagnosis and sites of accumulation and demonstration and counts of muscle fibers invaded by T cells," Annals of Neurology, vol. 16, no. 2, pp. 193-208, 1984.

[52] C. G. Bien, A. Vincent, M. H. Barnett et al., "Immunopathology of autoantibody-associated encephalitides: clues for pathogenesis," Brain, vol. 135, part 5, pp. 1622-1638, 2012.

[53] F. Bernal, S. Shams'Ili, I. Rojas et al., "Anti-Tr antibodies as markers of paraneoplastic cerebellar degeneration and Hodgkin's disease," Neurology, vol. 60, no. 2, pp. 230-234, 2003.

[54] P. Demaerel, W. Van Dessel, W. Van Paesschen, R. Vandenberghe, K. Van Laere, and J. Linn, "Autoimmune-mediated encephalitis," Neuroradiology, vol. 53, no. 11, pp. 837-851, 2011.

[55] T. Iizuka, S. Yoshii, S. Kan et al., "Reversible brain atrophy in anti-NMDA receptor encephalitis: a long-term observational study." Journal of neurology, vol. 257, no. 10, pp. 1686-1691, 2010.

[56] S. E. Monstad, A. Knudsen, H. B. Salvesen, J. H. Aarseth, and C. A. Vedeler, "Onconeural antibodies in sera from patients with various types of tumours.," Cancer Immunology, Immunother$a p y$, vol. 58, no. 11, pp. 1795-1800, 2009.

[57] F. Graus, F. Vega, J. Y. Delattre et al., "Plasmapheresis and antineoplastic treatment in CNS paraneoplastic syndromes with antineuronal autoantibodies," Neurology, vol. 42, no. 3, part 1, pp. 536-540, 1992.

[58] F. Blaes, M. Strittmatter, S. Merkelbach et al., "Intravenous immunoglobulins in the therapy of paraneoplastic neurological disorders," Journal of Neurology, vol. 246, no. 4, pp. 299-303, 1999.

[59] B. M. Ances, R. Vitaliani, R. A. Taylor et al., "Treatmentresponsive limbic encephalitis identified by neuropil antibodies: MRI and PET correlates," Brain, vol. 128, part 8, pp. 1764-1777, 2005.

[60] H. Ishiura, S. Matsuda, M. Higashihara et al., "Response of antinmda receptor encephalitis without tumor to immunotherapy including rituximab," Neurology, vol. 71, no. 23, pp. 1921-1923, 2008.

[61] J. Dalmau, F. Graus, A. Villarejo et al., "Clinical analysis of antiMa2-associated encephalitis," Brain, vol. 127, no. 8, pp. 18311844, 2004.

[62] A. Storstein, S. E. Monstad, M. Haugen et al., "Onconeural antibodies: improved detection and clinical correlations," Journal of Neuroimmunology, vol. 232, no. 1-2, pp. 166-170, 2011. 


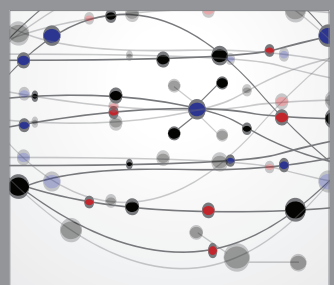

The Scientific World Journal
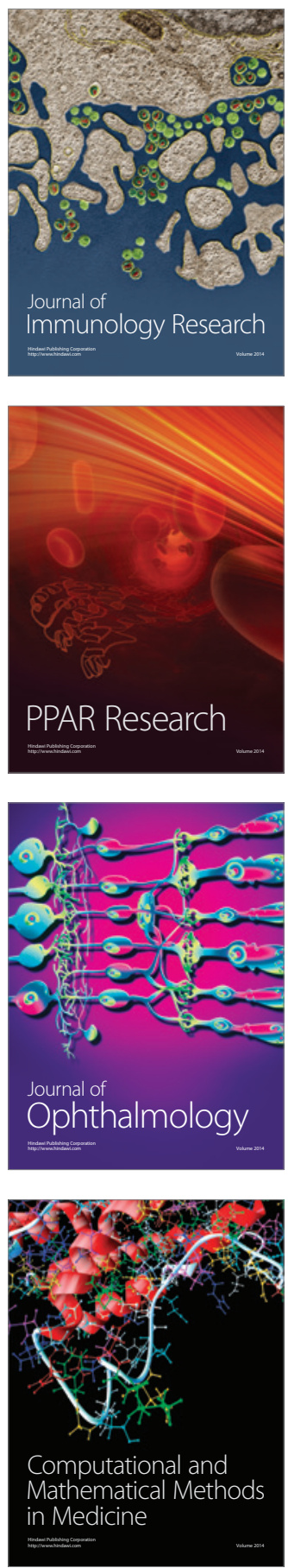

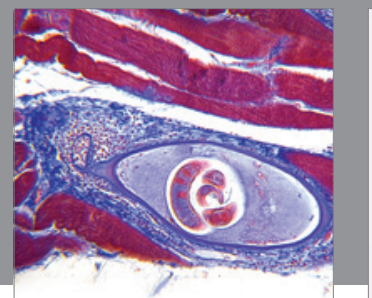

Gastroenterology

Research and Practice
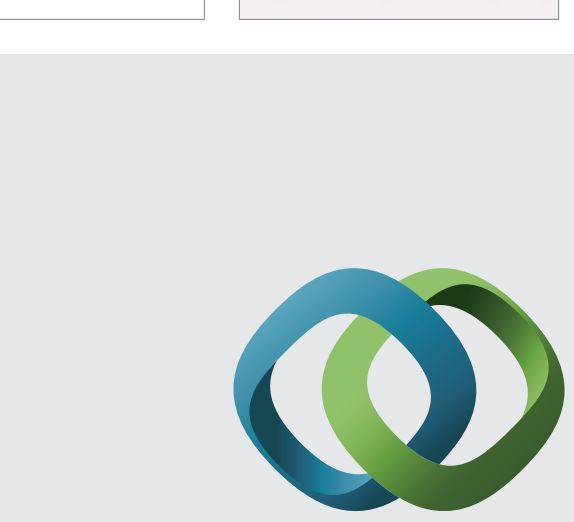

\section{Hindawi}

Submit your manuscripts at

http://www.hindawi.com
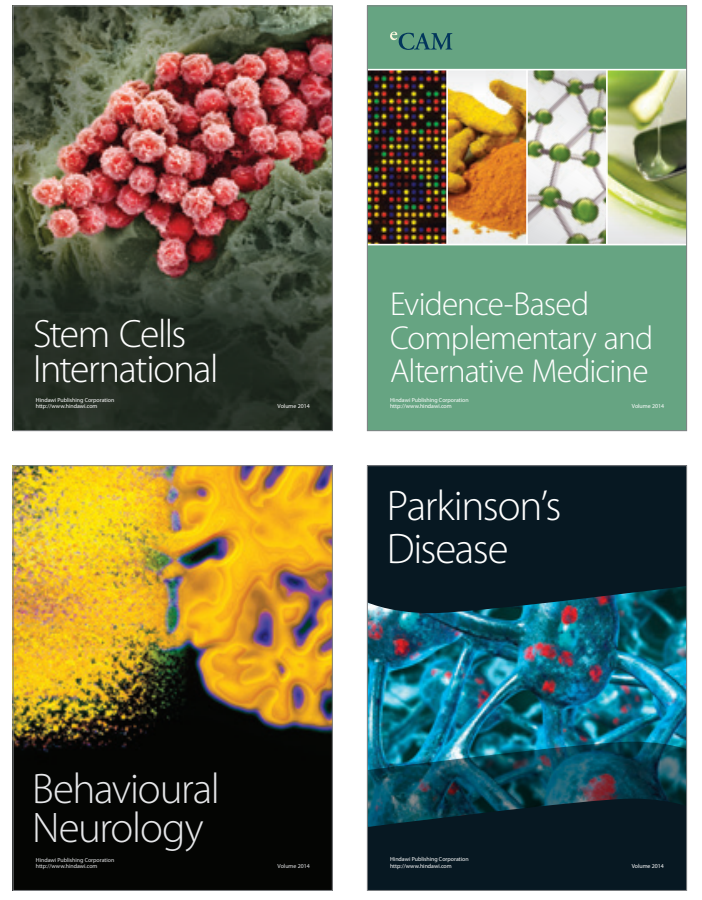
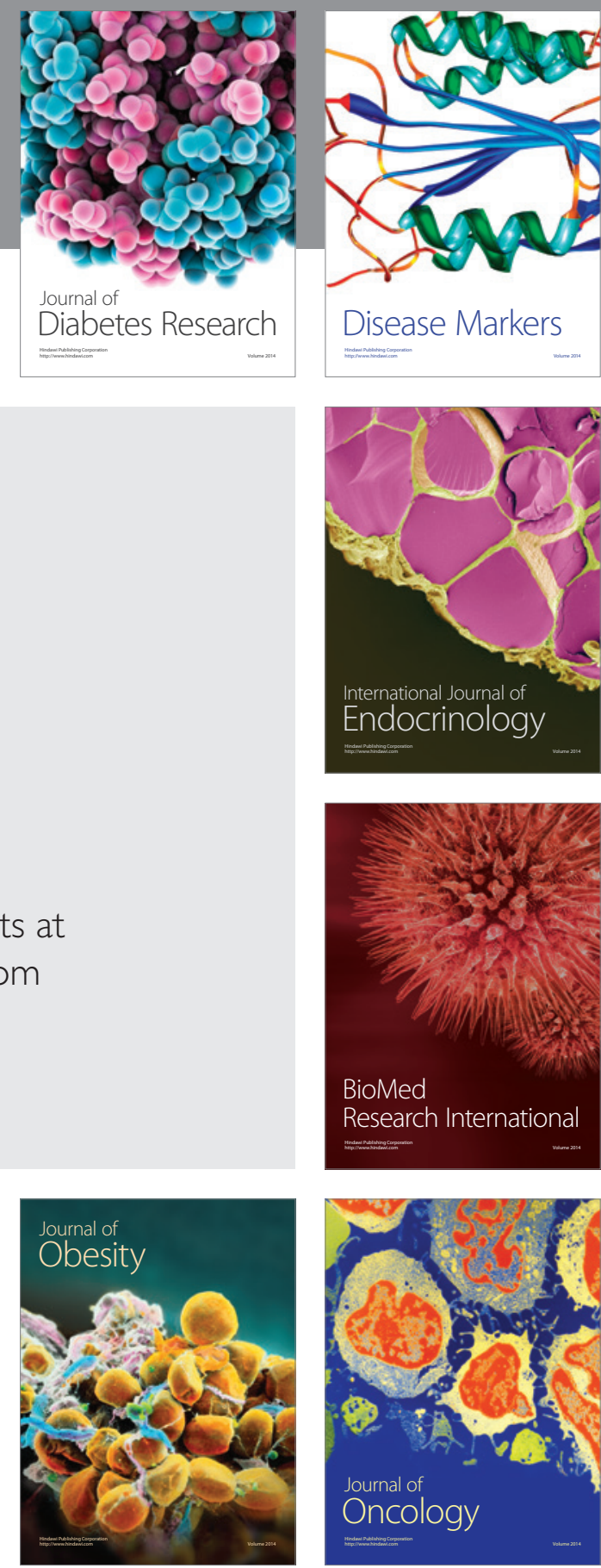

Disease Markers
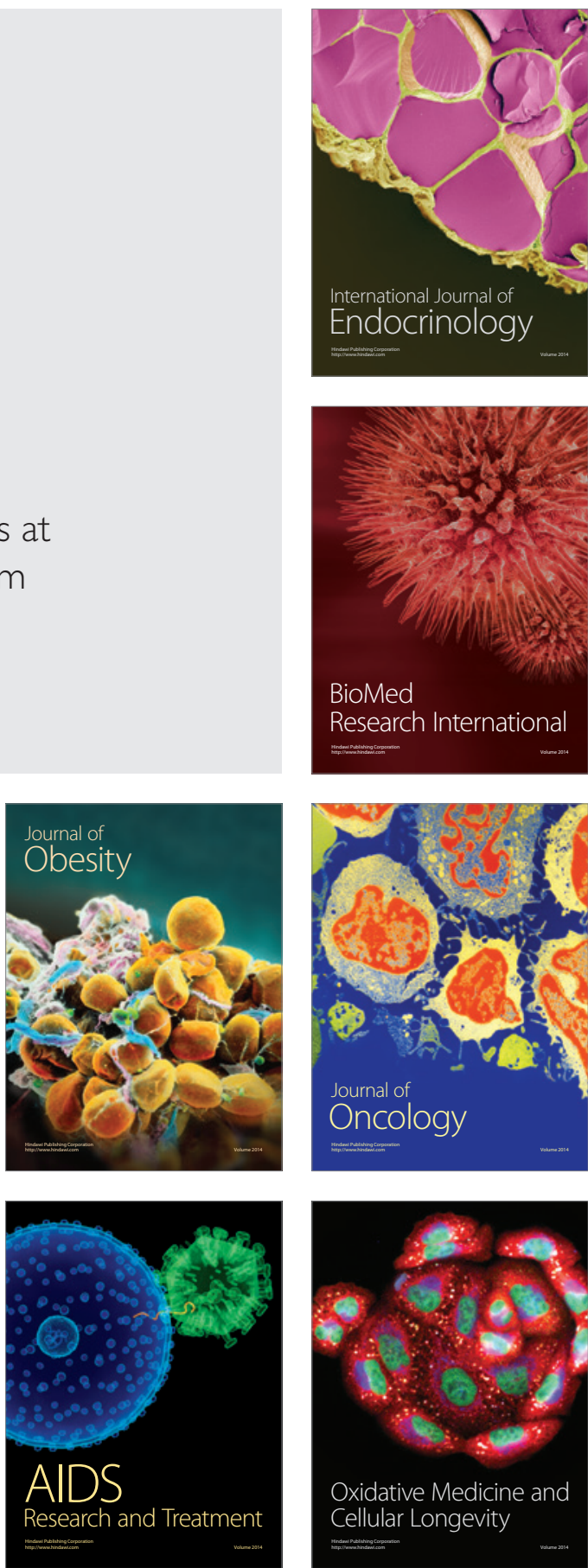